\title{
Added Value of Intraoperative Real-Time Imaging in Searches for Difficult-to-Locate Sentinel Nodes
}

Sergi Vidal-Sicart ${ }^{1,2}$, Pilar Paredes ${ }^{1}$, Gabriel Zanón ${ }^{3}$, Jaume Pahisa ${ }^{2,3}$, Sergio Martinez-Román ${ }^{3}$, Xavier Caparrós $^{3}$, Antoni Vilalta ${ }^{4}$, Ramon Rull ${ }^{5}$, and Francesca Pons ${ }^{1,2}$

${ }^{I}$ Nuclear Medicine Department (CDIC), Hospital Clínic Barcelona, Barcelona, Spain; ${ }^{2}$ Institut d'Investigacions Biomèdiques August Pi i Sunyer (IDIBAPS), Hospital Clínic Barcelona, Barcelona, Spain; ${ }^{3}$ Gynaecology Department (ICGON), Hospital Clínic Barcelona, Barcelona, Spain; ${ }^{4}$ Dermatology Department (ICMiD), Hospital Clínic Barcelona, Barcelona, Spain; and ${ }^{5}$ Surgery Department (ICMDM), Hospital Clínic Barcelona, Barcelona, Spain

Localization of sentinel lymph nodes can be challenging if they are in difficult anatomic locations or near high radiotracer activity. The purpose of this study was to assess the value of intraoperative real-time imaging using a portable $\gamma$-camera in conjunction with a conventional $\gamma$-counting probe when it is difficult to localize the sentinel node. Methods: After 99mTcnanocolloid injection, patients with various malignancies underwent presurgical lymphoscintigraphy followed by surgery (usually the next day). We evaluated 20 patients who required sentinel lymph node biopsy and in whom the location or other characteristics of the sentinel node would make intraoperative retrieval difficult. During surgery, the sentinel node was localized using a portable $\gamma$-camera together with a hand-held $\gamma$-probe. A ${ }^{153} \mathrm{Gd}$ pointer or ${ }^{125}$ I seed was used to better depict the sentinel node location in real time. Results: Using only a conventional hand-held $\gamma$-probe, surgeons were able to definitively localize the sentinel node in 15 of 20 patients. Intraoperatively, the portable $\gamma$-camera showed uptake by the definite sentinel node in 19 of 20 patients and helped to precisely localize the node with the hand-held $\gamma$-probe in 4 patients. In 1 of these patients, the sentinel node was metastatic. Conclusion: The combination of a standard hand-held $\gamma$-probe and real-time imaging provided by a portable $\gamma$-camera offers a high intraoperative detection rate in patients with difficult sentinel node localization as assessed by presurgical lymphoscintigraphy.

Key Words: portable gamma camera; sentinel node; real-time imaging; lymphoscintigraphy

J Nucl Med 2010; 51:1219-1225

DOI: 10.2967/jnumed.110.074880

$\mathbf{T}$ the 1990s as a staging technique replacing the then-stand ard procedure, complete regional lymphadenectomy. The new procedure was validated for malignancies such as mel-

Received Jan. 14, 2010; revision accepted Apr. 28, 2010.

For correspondence or reprints contact: Sergi Vidal-Sicart, Hospital

Clinic Barcelona, Villarroel 170, 9a ground floor, Barcelona 08036, Spain. E-mail: svidal@clinic.ub.es

COPYRIGHT $\odot 2010$ by the Society of Nuclear Medicine, Inc. anoma and breast cancer, came into routine clinical use for such malignancies, and is still the standard of care in their initial stages $(1-4)$.

Over time, as the technique evolved, this procedure came into use for other malignancies, such as penile, vulvar, and head and neck cancers. In all these tumors, lymphatic drainage can be characterized as predominantly superficial, and sentinel nodes can be successfully mapped by means of preoperative lymphoscintigraphy and the intraoperative use of a hand-held $\gamma$-probe or vital dye (5-7). However, the localization of sentinel nodes presents difficulties in some lymphatic basins of the neck in patients with head and neck cancer or melanoma. Also, sentinel nodes outside the axilla in breast cancer patients are sometimes difficult to retrieve. Although conventional lymphoscintigraphy plays a pivotal role in the depiction of drainage areas and a hand-held $\gamma$-probe is used to intraoperatively assess the location of the sentinel node, its position cannot always be clearly identified, potentially producing misleading results $(8,9)$.

New devices have been developed to deal with the limitations of planar lymphoscintigraphy and hand-held $\gamma$-probes. One important advance is the introduction of hybrid imaging devices such as SPECT/CT. SPECT/CT images, which clearly depict the sentinel nodes within an anatomic landscape, can provide a useful road map for surgeons $(10,11)$. Probably the most important contribution of SPECT/CT will be for those tumors with deeper drainage, such as gastrointestinal, gynecologic, and urologic malignancies (12).

Recently, there have been several reports on the use of portable $\gamma$-cameras in clinical and experimental settings (13-18). These devices play a remarkable role in the incorporation of imaging during surgery and can be combined with the information obtained preoperatively by lymphoscintigraphy or, even better, with information from previously performed SPECT/CT. Using the anatomic landmarks of SPECT/CT images, the portable device can be oriented to surgical targets in the operating room. Moreover, the possibility of obtaining a 
real-time image can be helpful to the surgeon, resulting in more reliable and, probably, briefer surgery, especially when the sentinel node is in a difficult anatomic area or near the radiotracer injection point (18-20).

The aim of the current study was to evaluate the use of a portable $\gamma$-camera during surgery. The included cases were rated difficult as judged by the nuclear physician and surgeon on the basis of the preoperative lymphoscintigraphy findings and the expected extended surgery time.

\section{MATERIALS AND METHODS}

\section{Patients}

From September 2008 to September 2009, we performed 210 sentinel lymph node procedures on melanoma, breast, and gynecologic cancers. Twenty of these cases had sentinel nodes that were rated as difficult to localize. This rating was based on the preoperative lymphoscintigraphy findings (weak uptake, nodes near the injection site, unclear locations, cluster of nodes), the nuclear physician's and surgeon's experience with the procedure, and their judgment of which cases might prove to require extended surgery time.

The data and characteristics of the 20 patients are listed in Table 1. Patients were included in this study if preoperative imaging showed a sentinel node. When no node was visualized during presurgical lymphoscintigraphy, patients were excluded from this evaluation. These patients had been diagnosed with malignant melanoma (5 patients), breast cancer ( 9 patients), and gynecologic cancer (6 patients).

\section{Presurgical Sentinel Node Localization}

All patients underwent preoperative lymphoscintigraphy after injection of $111 \mathrm{MBq}$ of ${ }^{99 \mathrm{~m} T c}$-nanocolloid (GE Healthcare). In melanoma and vulvar cancer patients, the tracer was injected intradermally, around the biopsy scar, in 4 deposits of $0.1 \mathrm{~mL}$ $(0.4 \mathrm{~mL}$ total $)$. In breast cancer patients, the tracer was administered intratumorally, in volumes of 0.5 or $0.3 \mathrm{~mL}$. The volume used depended on whether a tumor was palpable $(0.5 \mathrm{~mL})$ or nonpalpable $(0.3 \mathrm{~mL})$. In nonpalpable lesions, radiotracer was injected under ultrasonographic guidance. For gynecologic cancer patients, nanocolloidal particles were injected perilesionally. In cervical cancer patients, a volume of $2 \mathrm{~mL}$ was divided into 4 deposits of $0.5 \mathrm{~mL}$ each. In uterine cancer patients, the tracer was placed in anterior and posterior uterine walls, with transvaginal ultrasound guidance, in 2 deposits of $4 \mathrm{~mL}$ each ( $8 \mathrm{~mL}$ total).

After tracer injection, preoperative lymphoscintigraphy was performed on all patients. In patients with malignant melanoma, a dynamic study $(128 \times 128$ matrix, 20 images, 30 s/image $)$ was performed. Planar images were acquired in all cases at $30 \mathrm{~min}$ and $2 \mathrm{~h}$ after radiotracer injection. In some cases, a late image (4-6 h) was obtained.

Once delayed planar images were finished, SPECT $(128 \times 128$ matrix, 120 frames, $15 \mathrm{~s} /$ frame $)$ and low-dose CT $(512 \times 512$ matrix, $140 \mathrm{kV}, 2.5 \mathrm{mAs}$ ) were acquired, using a hybrid camera (Infinia Hawkeye 4; GE Healthcare).

After processing of raw images and correction for attenuation and scatter, corresponding SPECT and CT $4.5-\mathrm{mm}$ slices were generated in a Xeleris workstation (GE Healthcare). Images were fused using the appropriate workstation software and correctly analyzed using 2-dimensional reslicing in axial, coronal, and sagittal views.
From the scintigraphic point of view, sentinel nodes were those considered to be the first to appear in the dynamic study or in the sequential images in a specific lymph node basin, those directly connected with the injection zone by a lymphatic channel, or those meeting a combination of these criteria. Nodes appearing later in the same lymphatic stations were considered to be second-echelon nodes. If SPECT/CT demonstrated more hot spots in other areas with no other drainage or in zones closer to the injection site but without visualization on the planar images, those hot spots were also considered sentinel nodes. The location of the sentinel node was marked on the skin with an indelible-ink pen.

\section{Intrasurgical Sentinel Node Localization}

Surgery took place the day after the lymphoscintigraphic study, 18-20 $\mathrm{h}$ after tracer injection. Sentinel nodes were removed using the traditional approach in melanoma and breast cancer patients and laparoscopically in gynecologic cases, except for vulvar cancer.

For intraoperative sentinel node localization, a hand-held $\gamma$-probe (Navigator; USCC) was used (with a probe of 11-14 $\mathrm{mm}$ in cases of melanoma, breast cancer, and vulvar cancer and a laparoscopic probe of 11-mm diameter in cases of uterine and cervical cancer).

We used, in combination with the $\gamma$-probe, a portable $\gamma$-camera (Sentinella S102; GEM Imaging) fitted with a 4-mm pinhole collimator with a field of view of $4 \times 4 \mathrm{~cm}$. This field of view increases to $20 \times 20 \mathrm{~cm}$ when the camera is placed $18 \mathrm{~cm}$ from the patient's body.

In melanoma, breast cancer, and vulvar cancer, a ${ }^{153} \mathrm{Gd}$ pointer was used to better locate the sentinel node on the $\gamma$-camera screen. This pointer was introduced in a surgical glove, and during the operation the pointer was displayed separately (as a green circle) on the portable $\gamma$-camera screen. In laparoscopic procedures, we used as a pointer a ${ }^{125}$ I seed attached to the tip of a surgical grasper. The portable $\gamma$-camera was sterile-draped in such a way as to allow placement and movement above and within the surgical field. During each surgery, a first image of 60-120 s was acquired to assess the surgical field and validate sentinel node uptake. After incision, the hand-held $\gamma$-probe was introduced into the wound to locate the sentinel node. If there was any difficulty in finding the precise location of the sentinel node using the $\gamma$-probe (more than $5 \mathrm{~min}$ ), another image of 60-120 s was acquired using the portable $\gamma$-camera. Then, the surgeon placed the $\gamma$-camera above the previously marked sentinel node locations using the laser pointer fitted to the $\gamma$-camera and using the appropriate pointer depending on the case. The laser pointer is included in the supporting $\gamma$-camera structure and displays a red cross over the patient's skin. The position of this red cross is visible on the computer screen of the equipment. During surgery, the matching of 2 signals $\left({ }^{99 \mathrm{~m}} \mathrm{Tc}\right.$ signal and ${ }^{153} \mathrm{Gd}$ or ${ }^{125} \mathrm{I}$ pointer signals) showed the correct location of the sentinel nodes. This location was then checked using the $\gamma$-probe. After sentinel node retrieval, another set of images was acquired to ascertain the absence, or otherwise, of the previously visualized sentinel nodes or to ascertain the presence, or otherwise, of second-tier nodes.

All hot spots close to the marked areas were considered to be sentinel nodes. Second-echelon lymph nodes identified preoperatively were left in place. All sentinel nodes were sought using the hand-held $\gamma$-probe and the portable $\gamma$-camera and were removed wherever possible. All nodes were subsequently examined by expert pathologists. 
TABLE 1. Patient Characteristics and Results

\begin{tabular}{|c|c|c|c|c|c|c|}
\hline Patient no. & Age (y) & Location & Drainage & SN harvested & Time $(\min )^{*}$ & Pathology \\
\hline \multicolumn{7}{|l|}{ Melanoma } \\
\hline 1 & 50 & Parietal & Occipital/retro-SCM & 2 occipital, 1 retro-SCM & 10 & Negative \\
\hline 2 & 45 & Right lower limb & Popliteal/inguinal & 1 popliteal, 1 inguinal & 6 & Inguinal positive \\
\hline 3 & 71 & Nose & Submandibular & $\begin{array}{l}2 \text { left submandibular, } \\
2 \text { right submandibular }\end{array}$ & 10 & Negative \\
\hline 4 & 36 & Frontal & Parotid & $\begin{array}{l}2 \text { parotid, } 1 \text { preauricular, } \\
1 \mathrm{SCM}\end{array}$ & 10 & Negative \\
\hline 5 & 65 & Facial & Submandibular & 1 submandibular & 5 & Negative \\
\hline \multicolumn{7}{|l|}{ Breast cancer } \\
\hline 6 & 43 & Right upper outer & AX/IM & $1 \mathrm{AX}, 1 \mathrm{IM}$ & 10 & IM positive \\
\hline 7 & 66 & Left central lower & AX/IMC & $1 \mathrm{AX}, 1 \mathrm{IMC}$ & 10 & Negative \\
\hline 8 & 47 & Right upper inner & AX/IMC & $2 \mathrm{AX}, 2 \mathrm{IMC}$ & 20 & Negative \\
\hline 9 & 63 & Left upper innner & $\mathrm{AX} / \mathrm{IM}$ & $1 \mathrm{AX}, 1 \mathrm{IM}$ & 7 & AX positive \\
\hline 10 & 47 & Right upper inner & AX/IMC & $1 \mathrm{AX}, 1 \mathrm{IMC}$ & 12 & Negative \\
\hline 11 & 53 & Left upper inner & IMC & $1 \mathrm{IMC}$ & 10 & Negative \\
\hline 12 & 52 & Right lower outer & AX/IMC & $2 \mathrm{AX}, 1 \mathrm{IMC}$ & 10 & Negative \\
\hline 13 & 49 & Left central inner & $A X$ & $2 A X$ & 7 & $\mathrm{AX}_{\text {positive }}{ }^{\dagger}$ \\
\hline 14 & 56 & Left upper inner & $\mathrm{AX} / \mathrm{IMC}$ & $2 \mathrm{AX}, 2 \mathrm{IMC}$ & 14 & Negative \\
\hline \multicolumn{7}{|l|}{ Gynecologic } \\
\hline 15 & 43 & Cervix & Left pelvic & 1 external iliac & 4 & Negative \\
\hline 16 & 33 & Cervix & Bilateral pelvic & $\begin{array}{l}1 \text { right hypogastric, } 1 \text { left } \\
\text { external iliac }\end{array}$ & 7 & Negative \\
\hline 17 & 69 & Endometrium & Right pelvic & 1 obturator, 1 iliac & 10 & Obturator positive \\
\hline 18 & 37 & Cervix & Right pelvic & 1 obturator, 1 parametrial & 10 & Obturator positive \\
\hline 19 & 66 & Vulva (labia majora) & Bilateral inguinal & 2 right inguinal, 2 left obturator & 5 & Negative \\
\hline 20 & 34 & Endometrium & Right paraaortic & 2 precaval & 20 & Negative \\
\hline
\end{tabular}

\section{RESULTS}

Table 1 shows all sentinel nodes harvested, together with their pathologic results.

In the melanoma group, $4(80 \%)$ of 5 patients had a primary lesion in the head region, and the remaining patient had a lesion on a lower limb. Preoperative lymphoscintigraphy depicted at least 1 sentinel node in all cases. Two patients presented with a submandibular sentinel node, and 2 others showed a complicated drainage pattern with many nodes in occipital, preauricular, and cervical basins. Finally, the patient with the primary malignant melanoma on the lower limb presented with sentinel nodes in the popliteal and inguinal regions, as well as an in-transit node in the mid thigh (Fig. 1).

In this subgroup, when using only a hand-held $\gamma$-probe, surgeons were able to localize 12 sentinel nodes in 4 of 5 patients $(80 \%)$ but with some difficulties. In 1 patient, difficulty was caused by the high number of lymph nodes visualized on lymphoscintigraphy. In another patient, significant activity in the scar made localization difficult when only the probe was used. In this patient, an image acquired using the portable $\gamma$-camera helped facilitate excision of the sentinel node (Fig. 2).

When the portable $\gamma$-camera and the $\gamma$-probe were used together, surgeons were able to localize and excise addi- tional nodes assumed to be sentinel. Images acquired with the portable $\gamma$-camera were acquired in less than 2 min; 5 or fewer images were acquired in each case.

In the breast cancer group, of the 9 patients included in the study, all but 1 presented with drainage to axillary lymph nodes $(89 \%)$. Six patients presented with drainage in the inner mammary chain (67\%), and 2 showed intramammary sentinel nodes as well as axillary nodes (22\%).

In this subgroup, a total of 22 sentinel nodes were harvested: 12 axillary, 2 intramammary, and 8 in the inner mammary chain. Using only the hand-held $\gamma$-probe, surgeons correctly localized at least 1 sentinel node in each patient. However, they did not find all nodes considered sentinel in 2 (22\%) of 9 patients. Sentinel node localization with the $\gamma$-probe alone was not totally successful in a patient determined to have an intramammary node (Fig. 2) and in another patient observed to have inner mammary chain nodes with weak uptake on preoperative lymphoscintigraphy. On the other hand, when using the portable $\gamma$-camera with the $\gamma$-probe, surgeons localized 22 sentinel nodes. The mean time for localizing and harvesting sentinel nodes was $10 \mathrm{~min}$ - that is, the time from which the surgeon decided that use of the counting probe alone was not sufficient and began using the portable $\gamma$-camera until the time at which sentinel node retrieval was finished. 
FIGURE 1. Patient with malignant melanoma on right heel. Preoperative lymphoscintigraphy showed lymphatic channels toward mid thigh and inguinal region. SPECT/CT images show superficial popliteal node (A); portable $\gamma$-camera was positioned above this area with laser pointer centered on skin mark (B), and these images clearly demonstrate popliteal uptake in vivo (C) and, after sentinel node resection, absence of activity in surgical field (D). SPECT/CT images

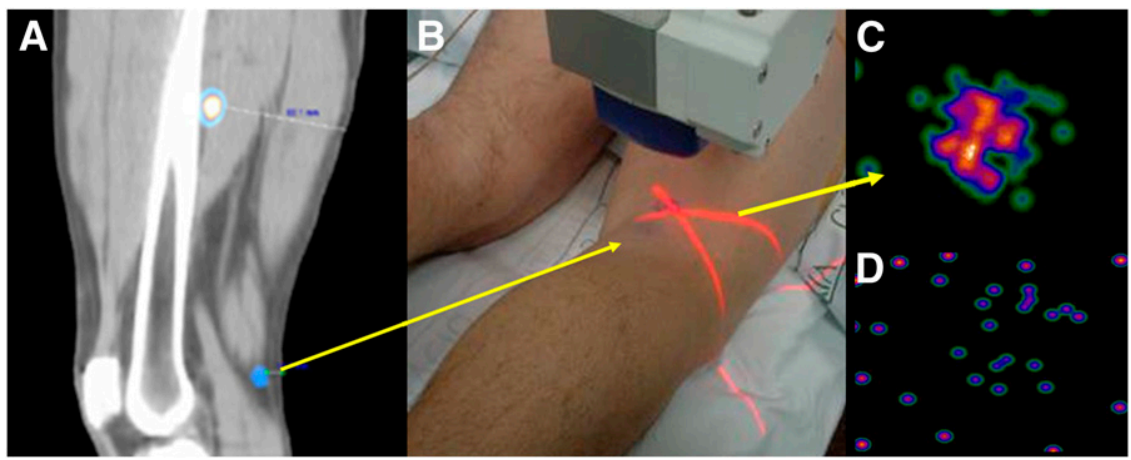
show deep localization of in-transit sentinel node in mid thigh. When portable $\gamma$-camera was imaging this area, tracer uptake could be seen. It was decided not to retrieve this activity because of its depth and because popliteal sentinel node in same channel had been retrieved earlier.

Overall, surgeons localized 19 of 22 harvested nodes using only the hand-held $\gamma$-probe. The other 3 harvested nodes were localized using the portable $\gamma$-camera and the $\gamma$-probe. Pathologic assessment of the nodes showed metastatic involvement in 3 patients (33\%). One of these showed a 4-mm metastasis in the intramammary node localized by the portable $\gamma$-camera. No axillary lymphadenectomy was performed, because the axillary sentinel node was negative for metastasis.

The gynecologic malignancy group included 3 patients with cervical cancer, 2 with endometrial tumor, and 1 with vulvar melanoma (which we decided to include in this group although it was a malignant melanoma). The lymphatic drainage was pelvic in all cervical tumors, pelvic or paraaortic in patients with endometrial cancer, and inguinal in the patient with vulvar melanoma. Thirteen sentinel nodes were excised in these patients, and the hand-held laparoscopic and traditional $\gamma$-probe clearly identified 10 of them (77\%). The portable $\gamma$-camera showed definite sentinel node uptake in $12(92 \%)$ of these sentinel nodes and weak activity in the remaining one. The cases in which the handheld $\gamma$-probe had difficulty localizing the sentinel node were 1 right parametrial node close to the radiotracer administration point and 2 right precaval nodes that were masked by high liver activity (Fig. 3). The time needed to localize the lymph nodes was different between vulvar/cervical malignancies and endometrial tumors (5-7 $\mathrm{min}$ and $15 \mathrm{~min}$, respectively).

In summary, using only the hand-held $\gamma$-probe, surgeons localized the sentinel nodes harvested in $15(75 \%)$ of 20 patients. Surgeons using the portable $\gamma$-camera and the $\gamma$-probe localized the sentinel nodes harvested in 19 $(95 \%)$ of 20 patients. One node harvested after the use of the $\gamma$-camera was metastatic. This node might have been missed if the camera had not been available during surgery. The mean time required for localizing and harvesting sen-

FIGURE 2. (A-C) Patient with left mandibular melanoma seen on delayed image (A). During surgery, surgeon using hand-held $\gamma$-probe could not precisely assess sentinel node location because residual activity was high even though wide excision had been performed. Images obtained with portable $\gamma$-camera 10 $\mathrm{cm}$ from patient did not show sentinel node (B), but in images acquired with camera closer to selected area, sentinel node (arrow) could be distinguished from radiotracer area (C). (D-F) Breast cancer patient with lymphatic drainage to intramammary and axillary nodes seen on preoperative image (D). Axillary sentinel node was harvested without difficulty with aid of hand-held $\gamma$-probe.
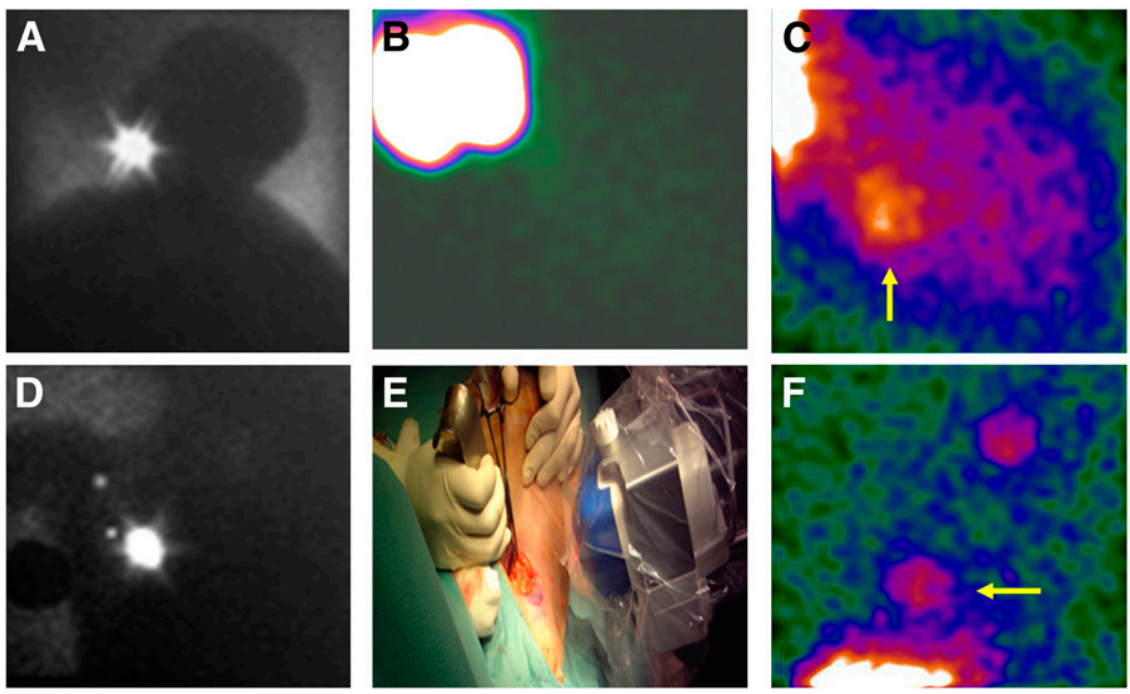
However, intramammary node could not be precisely located because of level of activity from primary tumor. Images obtained with real-time $\gamma$-camera (E) better depicted location of node (arrow) (F). 
tinel nodes with the portable $\gamma$-camera was 10 min (range, 4-20 $\mathrm{min})$.

\section{DISCUSSION}

Sentinel lymph node localization is a minimally invasive procedure, but in some circumstances the search for and removal of the correct node is challenging and timeconsuming (9).

This staging procedure has gained widespread use in melanoma and breast cancer and is currently being explored in many other malignancies (such as gynecologic and urologic cancers) (21). Although the reported success of sentinel node localization is high in most published studies, some node locations preclude a quick and easy retrieval. These cases require longer than the usual surgical time for localization and excision $(22,23)$.

Several studies have demonstrated the complexity and difficulty of excising head and neck sentinel nodes, with an intraoperative excision rate ranging from $75 \%$ to $100 \%$ (24-26). Another difficult aspect can be retrieval of intransit sentinel nodes, which in some circumstances appear in rare locations $(22,27)$. In all our patients in this setting, sentinel nodes could be retrieved without much difficulty by combining the hand-held $\gamma$-probe with the guidance and monitoring of the portable $\gamma$-camera. However, when only the probe was used, difficulties were experienced in 2 patients, one because of the presence of several nodes and the other because of the high activity remaining after
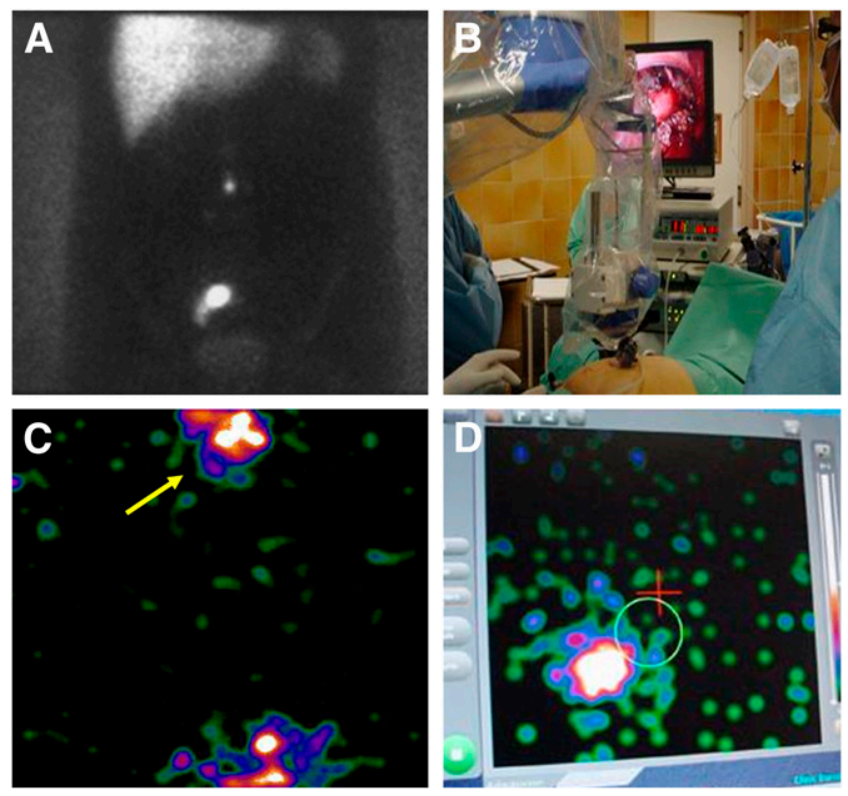

FIGURE 3. Patient with endometrial carcinoma and drainage to paraaortic region seen on preoperative image (A). During surgery, detection of sentinel node with handheld $\gamma$-probe was hampered by liver activity. Portable $\gamma$-camera was used to locate node (B). Portable $\gamma$-camera real-time images $(C)$ were definitive in retrieval of this node (arrow) by matching ${ }^{125}$ I seed (circle) with node activity (D). wide local excision. These kinds of complications are known pitfalls in the interpretation of presurgical lymphoscintigraphy and in intraoperative localization. The surgical time used to localize and harvest the sentinel nodes in this group averaged $10 \mathrm{~min}$.

In breast cancer patients, the situation can be especially difficult when intramammary or inner mammary chain nodes are observed or when lymph nodes are near the injection site (28-30). Most of these situations can now be resolved by a presurgical lymphoscintigraphic study with the aid of SPECT/CT (31). However, in the operating room, the method based on blue dye or the hand-held $\gamma$-probe can be misleading because of an absence of blue coloration, the presence of only faint uptake of tracer, or the presence of significant emission and scatter from the injection site. We encountered all these difficulties in our group of patients. In some, we were unable to adequately depict all sentinel nodes using only the hand-held $\gamma$-probe in difficult scenarios such as when one or more sentinel nodes were in intramammary or inner mammary chains. Real-time images acquired with the portable $\gamma$-camera were successfully used to find sentinel nodes that had been missed when only the $\gamma$-probe was used. Importantly, 1 node not found using the probe alone was positive for metastasis, and although no axillary lymphadenectomy was performed, this node was determinant in staging the disease. Mathelin et al. (32) evaluated the performance of an intraoperative $\gamma$-camera in the detection of axillary drainage and the assessment of sentinel node removal in 25 breast cancer patients. In 1 patient, this device allowed the detection of a residual sentinel node with low activity, which was massively metastatic. Soluri et al. (33) showed good results with a portable $\gamma$-camera in 30 breast cancer patients, with the advantage of an intraoperative-time reduction of $7 \mathrm{~min}$, and surgeons considered the use of the portable $\gamma$-camera very useful in 8 of 30 patients.

A high percentage of medial-quadrant tumors drain to the inner mammary chain, and hand-held probes cannot discriminate tumor activity from activity emitted by a tiny node in a deep intercostal region or behind a rib $(34,35)$. The possibility of identifying this space is a real advantage to the use of a portable $\gamma$-camera over the use of only a $\gamma$-counting probe, as intraoperative images can depict with reasonable precision the intercostal localization of a sentinel node.

Reported rates of intraoperative sentinel node identification are excellent in vulvar cancer and quite good in cervical cancer, ranging from $75 \%$ to $100 \%$ (36). Sentinel node identification in endometrial cancer shows wide variation due to a lack of standardization of the procedure, with localization rates ranging from $50 \%$ to $100 \%$ (37). Intraoperative difficulties are similar in the deep-drainage cancers, such as cervical and uterine cancers, in which sentinel nodes are near the injection site (parametrial nodes in cervical cancer) or outside the normal lymphadenectomy area (paraaortic nodes) (38). 
In our series, there were no significant differences between the $\gamma$-probe and the $\gamma$-camera in vulvar cancer. The portable $\gamma$-camera was used to monitor the pelvic area before and after removal of the hot nodes, to assess whether the sentinel node had been removed and to determine whether additional nodes or only background activity remained. This information is especially important in laparoscopic procedures, in which spatial mobility is limited and probe handling is not always easy. In this sense, Vermeeren et al. (39) showed the feasibility of using a portable $\gamma$-camera in urologic malignancies in 20 patients, with intraoperative identification in $90 \%$ of the patients, and raised the possibility of extending its use to other deep-draining malignancies.

Finally, the additional acquisition of several real-time images implies that surgical time must accommodate imaging time. In our study, the time for imaging may or may not be an actual addition to the surgical time, relative to a procedure in which no portable camera is used. If the portable camera is not used, a surgeon may need to spend a significant amount of time seeking additional nodes or confirming that no more are likely to be found. However, even if additional time is needed, our short series suggests that this extra time may have sufficient usefulness in the context of sentinel node procedures that are likely to be difficult, as the use of the $\gamma$-camera might reduce the possibility of missing a malignant sentinel node.

We are aware that this limited study describes the most complicated scenarios that can be observed when using the traditional approach with a hand-held $\gamma$-probe for sentinel lymph node localization. The current possibility of using tomographic and fused images preoperatively offers valuable information to the surgeon for the planning of appropriate surgery in each case. The introduction of a portable $\gamma$-camera for intraoperative guidance and real-time imaging is the next step to refining, as much as possible, the feasibility of the sentinel node technique in most tumors reported.

\section{CONCLUSION}

Our results in this particular series suggest that real-time imaging with a portable $\gamma$-camera in combination with a traditional $\gamma$-counting probe results in high detection and localization of sentinel nodes in cases for which such localization is expected to be difficult as determined through presurgical lymphoscintigraphy. Use of both technologies appears to add 10-15 min to the surgical time - an addition that we consider appropriate in view of the associated improvement in sentinel node retrieval.

\section{ACKNOWLEDGMENT}

This work was supported in part by Instituto de Salud Carlos III, Red Temática de Investigación Cooperativa en Cáncer (RTICC) RD06/0020/0038.

\section{REFERENCES}

1. Keshtgar MRS, Ell PJ. Sentinel lymph node detection and imaging. Eur J Nucl Med. 1999;26:57-67.

2. Gershenwald JE, Thompson W, Mansfield PF, et al. Multi-institutional melanoma lymphatic mapping experience: the prognostic value of sentinel lymph node status in 612 stage I or II melanoma patients. J Clin Oncol. 1999;17:976-983.

3. Veronesi U, Paganelli G, Viale G, et al. A randomized comparison of sentinelnode biopsy with routine axillary dissection in breast cancer. $N$ Engl J Med. 2003;349:546-553.

4. Morton DL, Cochran AJ, Thompson JF, et al. Sentinel node biopsy for earlystage melanoma: accuracy and morbidity in MSLT-I, an international multicenter trial. Ann Surg. 2005;242:302-313.

5. Kroon BK, Horenblas S, Nieweg OE. Contemporary management of penile squamous cell carcinoma. J Surg Oncol. 2005;89:43-50.

6. Vidal-Sicart S, Puig-Tintoré LM, Lejárcegui JA, et al. Validation and application of the sentinel lymph node concept in malignant vulvar tumours. Eur J Nucl Med Mol Imaging. 2007;34:384-391.

7. Povoski SP, Neff RI, Mojzisik CM, et al. A comprehensive overview of radioguided surgery using a gamma detection probe technology. World J Surg Oncol. 2009;7:11-74.

8. Tanis PJ, Nieweg OE, Van der Brekel M, Balm A. Dilemma of clinically nodenegative head and neck melanoma: outcome of "watch and wait" policy, elective lymph node dissection, and sentinel node biopsy-a systematic review. Head Neck. 2008;30:380-389.

9. Chakera AH, Friis E, Hesse U, Al-Suliman N, Zerahn B, Hesse B. Factors of importance for scintigraphic non-visualisation of sentinel nodes in breast cancer. Eur J Nucl Med Mol Imaging. 2005;32:286-293.

10. van der Ploeg IM, Valdes-Olmos RA, Nieweg OE, Rutger ETJ, Kroon BRB, Hoefnagel CA. The additional value of SPECT/CT in lymphatic mapping in breast cancer and melanoma. J Nucl Med. 2007;48:1756-1760.

11. Mucientes J, Cardona J, Delgado R, et al. SPECT-CT in sentinel node detection in patients with melanoma. Rev Esp Med Nucl. 2009;28:229-234.

12. Olmos RA, Vidal-Sicart S, Nieweg O. SPECT-CT and real-time intraoperative imaging: new tools for sentinel node localization and radioguided surgery? Eur J Nucl Med Mol Imaging. 2009;36:1-5.

13. Schillaci O, D'Errico G, Scafe R, et al. Sentinel node detection with imaging probe. Tumori. 2002;88(suppl):S32-S35.

14. Campisi C, Soluri A, Stella S, Valenti G, Scopinaro F. Intraoperative sentinel node detection by a innovative imaging probe. Tumori. 2002;88:S56-S58.

15. Pitre S, Ménard L, Ricard M, Solal M, Garbay JR. Charon Y. A hand-held imaging probe for radio-guided surgery: physical performance and preliminary clinical experience. Eur J Nucl Med Mol Imaging. 2003;30:339-343.

16. Aarsvod JN, Greene CM, Mintzer RA, et al. Intraoperative gamma imaging of axillary sentinel lymph nodes in breast cancer patients. Phys Med. 2006;21(suppl 1):76-79.

17. Tsuchimochi M, Hayama K, Oda T, Togashi M, Sakahara H. Evaluation of the efficacy of a small CdTe gamma-camera for sentinel lymph node biopsy. $\mathrm{J}$ Nucl Med. 2008;49:956-962.

18. Mathelin C, Salvador S, Croce S, Andriamisandratsoa N, Huss D, Guyonnet JL. Optimization of sentinel node biopsy in breast cancer using an operative gamma camera. World J Surg Oncol. 2007;5:132.

19. Paredes P, Vidal-Sicart S, Zanon G, et al. Radioguided occult lesion localisation in breast cancer using an intraoperative portable gamma camera: first results. Eur J Nucl Med Mol Imaging. 2008;35:230-235.

20. Mathelin C, Salvador S, Huss D, Guyonnet L. Precise localization of sentinel lymph nodes and estimation of their depth using a prototype intraoperative mini gamma-camera in patients with breast cancer. J Nucl Med. 2007;48:623-629.

21. Adib T, Barton DPJ. The sentinel lymph node: relevance in gynaecological cancers. Eur J Surg Oncol. 2006;32:866-874.

22. Silverio AM, McRae MC, Ariyan S, Narayan D. Management of the difficult sentinel lymph node in patients with primary cutaneous melanoma. Ann Plast Surg. 2009;62:564-569.

23. Liu LC, Lang JE, Jenkins T, et al. Is it necessary to harvest additional lymph nodes after resection of the most radioactive sentinel lymph node in breast cancer? J Am Coll Surg. 2008;207:853-858.

24. Reynolds HM, Smith NP, Uren RF, Thompson JF, Dunbar PR. Threedimensional visualization of skin lymphatic drainage patterns of the head and neck. Head Neck. 2009;31:1316-1325.

25. Chakera AH, Hesse B, Burak Z, et al. EANM-EORT general recommendations for sentinel node diagnostics in melanoma. Eur J Nucl Med Mol Imaging. 2009;36:1713-1742.

26. Doting EH, de Vries M, Plukker JT, et al. Does sentinel lymph node biopsy in cutaneous head and neck melanoma alter disease outcome? J Surg Oncol. 2006;93:564-570. 
27. Ortín-Perez J, Vidal-Sicart S, Domenech B, Rubí S, Lafuente S, Pons F. In-transit sentinel lymph nodes in malignant melanoma: what is their importance? Rev Esp Med Nucl. 2008;27:424-429.

28. Ogasawara Y, Yoshitomi S, Sato S, Doihara H. Clinical significance of preoperative lymphoscintigraphy for sentinel lymph node biopsy in breast cancer. J Surg Res. 2008;148:191-196.

29. Clímaco F, Coelho-Oliveira A, Djahjah MC, et al. Sentinel lymph node identification in breast cancer: a comparison study of deep versus superficial injection of radiopharmaceutical. Nucl Med Commun. 2009;30:525-532.

30. Tanis PJ, Deurloo EE, Valdés Olmos RA, et al. Single intralesional tracer dose for radio-guided excision of clinically occult breast cancer and sentinel node. Ann Surg Oncol. 2001;8:850-855.

31. Vermeeren L, van der Ploeg IM, Valdes-Olmos RA, et al. SPECT/CT for preoperative sentinel node localization. J Surg Oncol. 2010;101:184-190.

32. Mathelin C, Salvador S, Bekaert V, et al. A new intraoperative gamma camera for the sentinel lymph node procedure in breast cancer. Anticancer Res. 2008;28:2859-2864.

33. Soluri A, Massari R, Trotta C, et al. Small field-of-view, high-resolution, portable gamma camera for axillary sentinel node detection. Nucl Instrum Methods Phys Res A. 2006;569:273-276.
34. Hogan BV, Peter MB, Shenoy H, Horgan K, Shaaban A. Intramammary lymph node metastasis predicts poorer survival in breast cancer patients. Surg Oncol. 2010;19:11-16.

35. Madsen E, Gobardhan P, Bongers V, et al. The impact on post-surgical treatment of sentinel lymph node biopsy of internal mammary lymph nodes in patients with breast cancer. Ann Surg Oncol. 2007;14:1486-1492.

36. Roca I, Caresia P, Gil-Moreno A, et al. Usefulness of sentinel lymph node detection in early stages of cervical cancer. Eur J Nucl Med Mol Imaging. 2005;32:1210-1216.

37. Khoury-Collado F, Abu-Rustum NR. Lymphatic mapping in endometrial cancer: a literature review of current techniques and results. Int J Gynecol Cancer. 2008; 18:1163-1168.

38. Levenback CF, van der Zee AG, Rob L, et al. Sentinel lymph node biopsy in patients with gynecologic cancers: expert panel statement from the International Sentinel Node Society Meeting, February 21, 2008. Gynecol Oncol. 2009;114: $151-156$.

39. Vermeeren L, Valdes-Olmos RA, Meinhartd W, et al. Intraoperative radioguidance with a portable gamma camera: a novel technique for laparoscopic sentinel node localisation in urological malignancies. Eur J Nucl Med Mol Imaging. 2009; 36:1029-1036. 